Moniek van der Zanden*, Laura de Kok, Willianne L. D. M. Nelen, Didi D. M. Braat and Annemiek W. Nap

\title{
Strengths and weaknesses in the diagnostic process of endometriosis from the patients' perspective: a focus group study
}

https://doi.org/10.1515/dx-2021-0043

Received March 25, 2021; accepted June 17, 2021;

published online July 28, 2021

\section{Abstract}

Objectives: Endometriosis is characterized by a long interval between onset of symptoms and diagnosis. Detailed information about the patients' perspective on the diagnostic delay of endometriosis is scarce. The aim of this study was to identify strengths and weaknesses in the diagnostic process of endometriosis from the patients' perspective.

Methods: Qualitative study with semi-structured focus groups. Participants were women between 18 and 45 years old with a recently confirmed diagnosis. The focus groups were audio recorded and fully transcribed. Grounded theory methodology was applied for data analysis.

Results: Six focus groups were organized, in which 23 women participated. Mean time from start of symptoms to diagnosis was 8.5 years. Dominant themes were knowledge about normal menstruation and endometriosis, being believed and acknowledged by medical staff, and collaboration between health care providers including fast referral to a gynaecologist. Barriers to a timely referral and diagnosis were young age, normalization of symptoms and a lack of awareness in general practitioners. Facilitating factors included adequate knowledge and skills of the general practitioner, a desire for pregnancy and persisting in a request for specialist consultation by the patient.

\footnotetext{
*Corresponding author: Moniek van der Zanden, MD, PhD, Department of Gynecology and Obstetrics, Radboud University Medical Center, P.O. Box 9101, 6500 HB, Nijmegen, The Netherlands, Phone: +31243614725 , E-mail: Moniek.vanderzanden@radboudumc.nl Laura de Kok, Willianne L. D. M. Nelen and Didi D. M. Braat, Department of Gynecology and Obstetrics, Radboud University Medical Center, Nijmegen, The Netherlands

Annemiek W. Nap, Department of Gynecology and Obstetrics, Radboud University Medical Center, Nijmegen, The Netherlands; and Department of Gynecology and Obstetrics, Rijnstate Hospital, Arnhem, The Netherlands
}

Conclusions: The diagnostic process of endometriosis is hampered by delayed consultation, inadequate appraisal of symptoms by general practitioners and the interaction between patients and medical professionals. Efforts should be made to increase public awareness and to provide medical staff with sufficient knowledge and skills to adequately acknowledge presented symptoms.

Keywords: diagnostic delay; endometriosis; focus group; patients perspective; qualitative study.

\section{Introduction}

Endometriosis is one of the most prevalent gynecologic conditions in women of reproductive age, and the most common cause of chronic pelvic pain [1-3]. Symptoms related to endometriosis commonly include dysmenorrhea, pelvic pain, dyspareunia and infertility but a wide spectrum of physical symptoms may be involved [3, 4]. Women with endometriosis experience a decreased quality of life, and considerable loss of work productivity with associated costs have been identified [5-8]. Unfortunately, endometriosis is characterized by a long interval between onset of symptoms and diagnosis [6, 9-12]. This diagnostic delay is partly due to factors related to the condition itself, like the variable symptom pattern, whether or not subfertility is present, and the fact that invasive diagnostic testing using laparoscopy has been the gold standard for diagnosis [9, 13]. Factors related to the health care organization, the patients' help seeking behavior and medical professionals' clinical strategies including referral habits have been proven of great importance as well [9, 10, 12, 14]. Research concerning endometriosis tends to focus on pathogenesis and medical strategies for diagnosis and treatment. However, recent studies on quality of care in endometriosis have shown increased interest in patient-centeredness [15-19]. A systematic review on patient-centeredness of endometriosis care revealed that the most frequently reported improvement targets were 'timely diagnosis' and 'being believed and respected by medical professionals [15]. 
Although several studies have addressed these items $[9,10,12,20,21]$, more in-depth information regarding delays in diagnosing endometriosis from the patients' perspective is needed to propose relevant interventions for improvement. The aim of this study was to identify strengths and weaknesses in the current diagnostic process of endometriosis from the patients' perspective, covering both their own help seeking behavior and their experience with medical professionals on their journey towards the diagnosis of endometriosis.

\section{Materials and methods}

\author{
Study design and data collection
}

Focus group interviews with women between 18 and 45 years old, who have been diagnosed with endometriosis in the previous five years, have been conducted between March 2016 and March 2017. Participants were recruited by advertisements in social media, a national patient interest group and centers of expertise in endometriosis. The focus groups were organized throughout the country in conference rooms. Semi-structured interviews in groups of two to six participants were carried out rather than individual interviews because we expected that the interaction and sharing of experiences would generate more relevant information. The semi-structured approach allowed the participants to talk freely with structured guidance from the moderator, using an interview guide (see Supplementary material). The interview guide was based on the literature and experience of the authors, working in the fields of reproductive medicine, qualitative research, and implementation research. The topic guide was a dynamic document, on which topics were added when new items were identified during the interviews. In addition, each participant was asked to complete a short questionnaire to inform the researchers about their age, timeline in their diagnostic process and treatment history and personal suggestions for reducing the diagnostic delay. The group interviews were directed by a moderator (AN or MZ) with a backup for taking notes and process monitoring (MZ, LK or AN). We continued to organize focus group interviews until data saturation was achieved, which was defined as no additional information was gathered during subsequent focus group interviews, followed by one additional session for confirmation of data saturation. The participants received detailed information about the study design and signed an informed consent form prior to participating. Anonymity and confidentiality were ensured.

\section{Data analysis}

The focus group sessions were audio recorded and fully transcribed. The qualitative research software package ATLAS-ti (v7.1) was used to assist in data analysis. We used the grounded theory methodology for data analysis [22, 23]. First, all transcripts were analyzed by two of the authors (MZ and LK) independently by means of line by line open coding. After the individual coding of each transcript, quotation selection and codes were compared, and any discrepancies were resolved by discussion between the two researchers. Remaining differences of opinion were discussed with a third researcher (WN) until consensus was reached. This third researcher, a qualitative research specialist, checked samples of the transcripts for completeness of coding. After coding of all the transcripts, the full code list was analyzed by the three coding authors and the researchers who moderated the focus group interviews. In this session, the codes were grouped into categories and clustered into themes or domains. A summary of the analysis process is shown in Figure 1. Study reporting was based on the Consolidated Criteria for Reporting Qualitative Health Research (COREQ) [24]. We provided a quantification to indicate whether the results have been obtained from few (1-3), some (4-7), many (8-11) or most (12 or more) participants.

\section{Ethical approval}

The study protocol was evaluated by the Radboud University Medical Centre Research Ethics Committee and did not require full Institutional Review Board approval (Reference number 2016-2629).

\section{Results}

Six focus groups were conducted, in which in total 23 women participated. The duration of the focus groups was between 66 and $97 \mathrm{~min}$. Mean age of participants was 33.9 years (range 29-45). Mean time from start of symptoms to diagnosis was 101.7 months or 8.5 years (range 0-216 months, SD 81.3 months). Most of the delay consisted of the time from onset of symptoms to first visit to the general practitioner (33 months) and time between first general practitioner visit and referral to a gynaecologist (61 months). Mean time since diagnosis was 3.8 years (range $0.5-9$ years, SD 2.6 years). Two women were referred to the gynaecologist because of infertility, the other women had pelvic pain or other symptoms related to endometriosis. Twenty-one women (91.3\%) reported a laparoscopy or MRI for confirming the diagnosis of endometriosis; the other two were diagnosed by gynecologic examination including ultrasound imaging.

Analysis of the focus group interviews generated three main themes based on grouping of the most frequent codes and categories: (1) knowledge about normal menstruation and endometriosis in both the general population and medical professionals, (2) being believed and acknowledged by medical professionals, and (3) collaboration between health care providers including fast referral to a gynaecologist.

\section{Knowledge about normal menstruation and about endometriosis}

Many of the participating women reported problematic menstruation starting shortly after menarche or in adolescence. Help seeking behavior was predominantly influenced 


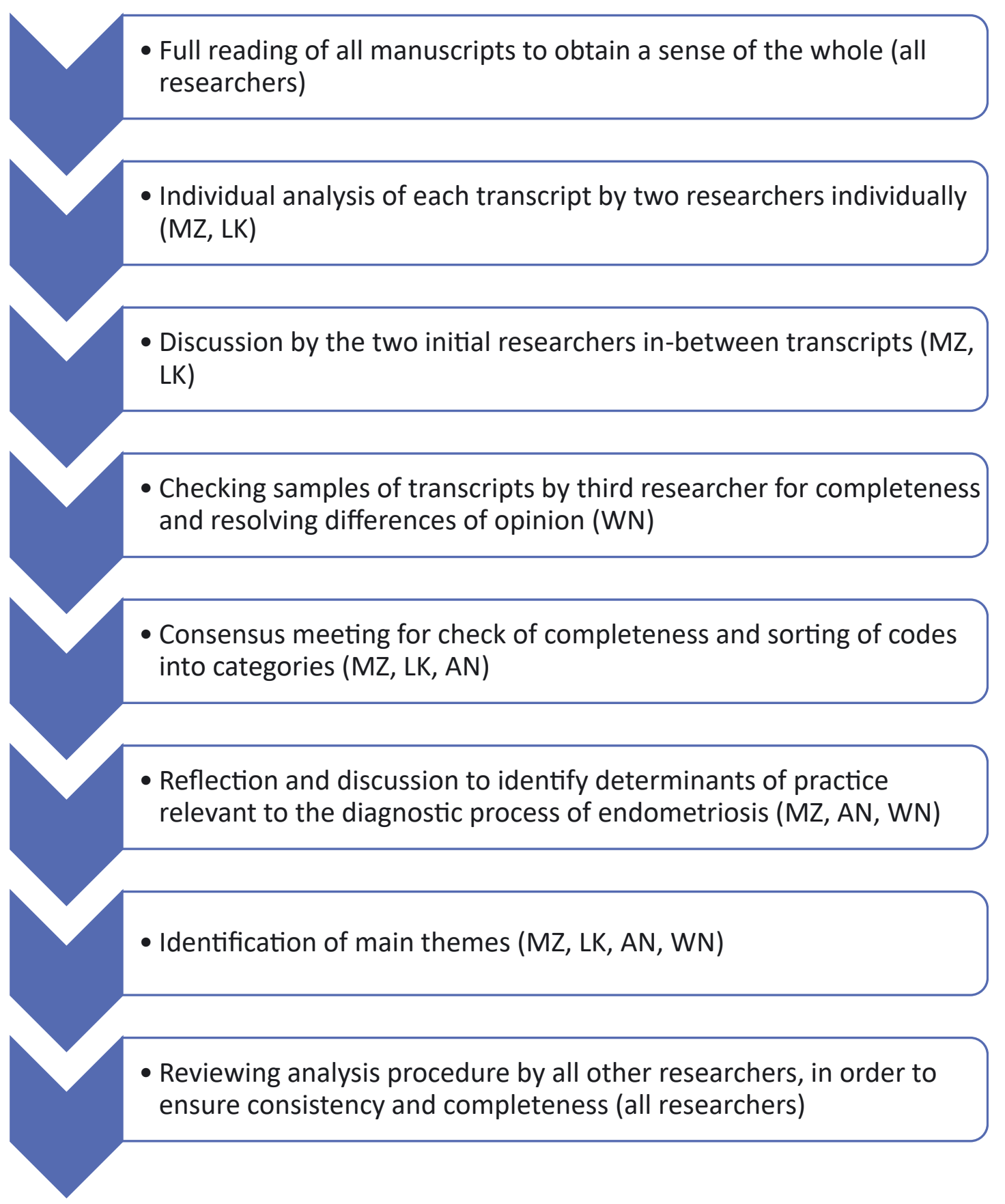

Figure 1: Summary of analysis process.

by the interpretation of these complaints not only by the women themselves, but also by relatives and friends, in particular the mother. If participants had been told that their complaints were within a normal range, or that they were just unlucky to have pain, they started to dissimilate as well. When their mother, or significant others supported them in their feeling something was wrong, they were more likely to consult a doctor for their complaints. Some women mentioned that they suspected their mother had suffered from endometriosis as well, based on comparison of symptoms, without ever being diagnosed. These women were more frequently told that they just had to learn to live with the pain or take pain medication.

My mom told me it was normal and that I was just unlucky. [31 y/o, delay 18 years]

A few women, who considered their complaints as normal, were directed to their general practitioner by a friend or relative who incidentally captured them during a painful period and told them it was not normal. Although many 
participants had compared their menstruation with their friends at young age, some also mentioned feelings of shame and perception of taboo.

I was on a trip to Greece with a friend. I was lying on a chair like I was dying, and she looked at me and said "what are you doing?" Then I told her I felt like this every month and we started to talk about it [menstruation] and then I realized it was not normal. I was 30 years old at the time. [M, $37 \mathrm{y} / \mathrm{o}$, delay 14 years]

\section{Being acknowledged by medical professionals}

Almost all women with pelvic pain reported repeated consultations at the general practitioner's office for a diversity of symptoms which could later be attributed to endometriosis. Almost all of these women felt their symptoms were normalized or not taken seriously by the general practitioner. They perceived a lack of knowledge and skills concerning endometriosis resulting in insufficient acknowledgment in their call for help. Some were submitted to non-discriminatory tests or referred to the wrong medical specialist or given numerous prescriptions for pain medication or contraceptive pills, often without the advice to take them continuously. Some of the participants never had a gynecologic examination by their general practitioner despite repetitive consultations for abdominal pain or problematic menstruation. Five participants experienced restraint in further testing or referral because they were too young. Others were referred to the gynaecologist only after seeing a different general practitioner or during an emergency hospitalization. The gender of the general practitioner did not seem to affect the evaluation of symptoms according to the participants.

My general practitioner never took it seriously because I didn't want to get pregnant yet. I was 15 or 16 years old ... I got prescribed different contraceptive pills all the time. I got referred when I was 20 years old and was trying to conceive. [H, 29 y/o, delay 10 years]

There were participants with a fast referral to a gynaecologist as well. This was more likely if the patients reported specific complaints related to the menstruation, if they had tried to conceive for more than one year, if the women suggested endometriosis themselves or if they persisted in requesting referral.

It was left in the middle for a while by the general practitioner. At some point I demanded a referral to a gynaecologist. My general practitioner thought it was ridiculous en unnecessary. But I persisted and then he said fine let's do it. [M, 37 y/o, delay 14 years]

\section{Collaboration between health care providers}

For most women, diagnosis was confirmed shortly after referral to a gynaecologist. Some women had multiple gynaecologist consultations before (complete) diagnosis, and participants observed an inequality in knowledge and skills between gynaecologists. A few women were diagnosed with endometriosis, but the full extent of the disease, in particular deep endometriosis, was not recognized from the beginning because of incomplete examination. Several participants encountered medical specialists other than gynaecologists because of their complaints, and many of them experienced that other specialists did not consider a gynecologic condition or there was insufficient communication and collaboration between specialists.

\begin{abstract}
I just wished an internal medicine specialist or a urologist would think of it more easily. You'd say they work in the same hospital, why don't you just call the gynaecologist to ask if they have any idea what is going on. [E, $30 \mathrm{y} / \mathrm{o}$, delay 11 years]
\end{abstract}

\section{Suggested interventions to reduce diagnostic delay}

The participants were asked about their suggestions to reduce diagnostic delay in endometriosis. Suggested interventions are given in Table 1. The most important interventions according to the participants were directed at increasing awareness in the general population and providing medical

Table 1: Interventions to reduce diagnostic delay as suggested by the participants.

\begin{tabular}{lr}
\hline $\begin{array}{l}\text { Suggested intervention to reduce diagnostic } \\
\text { delay }\end{array}$ & $\begin{array}{r}\text { Number of } \\
\text { participants }\end{array}$ \\
\hline $\begin{array}{l}\text { Improve knowledge about normal menstruation } \\
\text { and endometriosis, recognizing of symptoms }\end{array}$ & 13 \\
by GPs & 11 \\
Taking symptoms seriously and & 9 \\
acknowledgement & \\
More publicity about menstruation and endo- & 8 \\
metriosis in general & 6 \\
Improve knowledge in other medical specialists & 5 \\
and collaboration & 2 \\
Faster referral to gynaecologists by GPs & 1 \\
Improve quality of physical examination & 1 \\
Faster referral to specialized gynaecologist & \\
Multidisciplinary teams & 1 \\
Reduce waiting lists for centres of expertise in & \\
endometriosis & \\
No fertility treatment until complete diagnosis & \\
Improve knowledge in gynaecologists & \\
\hline
\end{tabular}


staff (in particular general practitioners) with sufficient knowledge and skills to interpret presented symptoms or clinical findings correctly. Again, being taken seriously and acknowledgement appeared of major importance.

\section{Discussion}

This qualitative study has yielded rich and important information about the experiences of women with endometriosis in reaching a diagnosis. The most striking finding was that most of the participants felt they were not taken seriously after disclosure of their symptoms. This influences their bodily perception and self-image, and may lead to delayed consultation and referral, especially if their mother or other loved ones do not acknowledge their symptoms correctly. Being taken seriously by the general practitioner was the most frequently used code to label citations from the transcripts, and the second most frequent suggestion for improvement by participants.

Although it was challenging to distinguish patterns or similarities given the unique and complex nature of the participants' social and medical histories, many women experienced comparable struggles in their journey towards diagnosis. We identified barriers and facilitators at three different levels: the women's personal level, knowledge and skills of medical professionals, in particular general practitioners, and the interaction between patients and their health care providers. With regard to the women's personal level, self-recognition and interference of significant others appeared crucial for seeking medical help. For young women, the opinion of their mother turned out to be of key importance for the appraisal of their symptoms. This is an important finding because it may determine time to first consultation. Factors influencing the shift from normalizing of symptoms to help seeking behavior have been proposed by Manderson et al. [25]. Of the four catalysts described in the latter study (intercession, social disruption, biographic disruption, and self-recognition), intercession and self-recognition were the most prevalent in our study population. Differentiating between normal and abnormal appeared equally difficult for general practitioners according to the participants. Most of the diagnostic delay in our study consisted of the time between first visit to the general practitioner and referral to a gynaecologist, which is in line with previous studies [9, 12]. The general practitioner needs to either recognize the presented symptoms as pathological or acknowledge the significant impact of symptoms on the patient's life and thereby justify referral for further diagnostic tests.
Diagnostic delay was estimated at 8.5 years in this study, which is comparable with the existing international literature $[9-12,26]$. In the Netherlands, the general practitioner has a strong position with regard to access to medical care, acting as a gatekeeper for medical specialist care. Other countries, including those in which specialist care is freely accessible, show a similar diagnostic delay $[9,10,26,27]$. We suggest that the first medical professional consulted is the medical professional encountering the problem of differentiating between 'normal' menstrual complaints and signs or symptoms suggestive of endometriosis. Failure of this process may lead to misdiagnosis and delayed diagnostic tests or treatment.

The importance of findings derived from qualitative research has been increasingly appreciated [22, 28]. However, careful consideration on study methodology is of great importance in order to secure study validity $[24,29$, 30]. To extract the most relevant information from the transcripts and preserve objectivity, we conducted a databased analysis style by two independent researchers, and all steps of data analysis were discussed with several members of the research team. A wide diversity of aspects relevant to the diagnostic process were discussed in the focus groups, including women's own appraisal of symptoms, key elements in seeking medical help, and experience with medical professionals, both in primary and secondary care. Some of the participants were treated at the clinic in which one of the researchers was employed. They were informed in advance, encouraged to speak freely, and ensured that their contribution to the discussion would not influence their treatment in any way. These focus groups were moderated by a researcher not employed in the particular clinic if known in advance.

Diagnostic delay in endometriosis has been subject of several studies in the past decades. Even though many contributing factors have been described, the diagnostic delay does not seem to diminish. When asked about their opinion about interventions to reduce the delay, the participants in our study mainly suggested improvements in knowledge and awareness and being taken seriously by medical professionals. They did not mention increasing efforts to expand scientific and medical knowledge, or focus on new diagnostic tests, but stressed the importance of being acknowledged in their symptoms and worries. Although the importance of developing an accurate noninvasive diagnostic test for endometriosis is obvious, in the meanwhile, it may be time to focus more on the clinical and personal aspects of reaching a diagnosis. Several studies suggested a switch from surgical to clinical diagnosis of endometriosis [31-33], a strategy that may reduce time between start of symptoms and adequate treatment even in 
the absence of a definite diagnosis. Focusing more on the person instead of the lesion creates opportunities for general practitioners to be more confident in their own diagnostic skills and start empiric treatment in a timely matter. Adequate education to provide general practitioners with more knowledge about specific signs, symptoms and clinical findings possibly related to endometriosis and indications for referral is warranted to increase awareness on the condition and ensure specialist care when needed. Implementing this strategy may decrease reluctance in general practitioners for referral because of fear of inappropriate invasive testing as seen in one of our previous studies [34]. It is of great importance to make sure that when empiric treatment is started, the presumed diagnosis of endometriosis is communicated with the patient, a standardized therapeutic algorithm is followed in which hormonal therapy is given continuously instead of intermittently, and short-term evaluation of the intervention is effectuated. In case of treatment failure or infertility, referral to a gynaecologist for specialist care (including expert medical treatment, advanced imaging, diagnostic or therapeutic laparoscopy and artificial reproductive techniques) is warranted. The development of a guideline, which is suitable for both general practitioners and gynaecologists or other medical specialists who encounter women in their reproductive life span with abdominal complaints, may support the feasibility of such a strategy. In addition, increasing awareness in the general population, in particular adolescent girls and women is of major importance to reduce patients' delay. Efforts have been made to identify the gaps in knowledge about endometriosis and preferences for information transmission in young women [35], and several countries including the United States and Australia have developed outreach programs to expand knowledge in adolescents [36]. The Dutch patient interest group on endometriosis recently reported on a possible increase in awareness on endometriosis in women, resulting in a reduction in time between onset of symptoms and seeking medical help [37]. These are important steps in reducing diagnostic delay in endometriosis.

\section{Conclusions}

In conclusion, the present study has shown that the diagnostic process in endometriosis is hampered by late presentation of symptoms to a medical professional, inadequate appraisal of symptoms and the interaction between patients and medical professionals. Women with endometriosis prompt their physicians to take them seriously. Efforts should be made to increase awareness in the general population, and to provide medical staff with sufficient knowledge and skills to adequately acknowledge presented symptoms or clinical findings and initiate appropriate actions.

Acknowledgments: The authors are grateful to the women who participated in this study for sharing their experiences. Research funding: None declared.

Author contributions: All authors have accepted responsibility for the entire content of this manuscript and approved its submission.

Competing interests: Authors state no conflict of interest. Informed consent: Informed consent was obtained from all individuals included in this study.

Ethical approval: The study protocol was evaluated by the Radboud University Medical Centre Research Ethics Committee and did not require full Institutional Review Board approval (Reference number 2016-2629).

\section{References}

1. Reese KA, Reddy S, Rock JA. Endometriosis in an adolescent population: the Emory experience. J Pediatr Adolesc Gynecol 1996;9:125-8.

2. Eskenazi B, Warner ML. Epidemiology of endometriosis. Obstet Gyn Clin Am 1997;24:235-58.

3. Bellelis P, Dias JA Jr, Podgaec S, Gonzales M, Baracat EC, Abrao MS. Epidemiological and clinical aspects of pelvic endometriosis-a case series. Rev Assoc Med Bras 1992 2010;56:467-71.

4. Davis GD, Thillet E, Lindemann J. Clinical characteristics of adolescent endometriosis. J Adolesc Health 1993;14:362-8.

5. Gao X, Yeh YC, Outley J, Simon J, Botteman M, Spalding J. Healthrelated quality of life burden of women with endometriosis: a literature review. Curr Med Res Opin 2006;22:1787-97.

6. Nnoaham KE, Hummelshoj L, Webster P, d'Hooghe T, de Cicco Nardone F, de Cicco Nardone C, et al. Impact of endometriosis on quality of life and work productivity: a multicenter study across ten countries. Fertil Steril 2011;96:366-73.

7. Fourquet J, Gao X, Zavala D, Orengo JC, Abac S, Ruiz A, et al. Patients' report on how endometriosis affects health, work, and daily life. Fertil Steril 2010;93:2424-8.

8. Simoens S, Dunselman G, Dirksen C, Hummelshoj L, Bokor A, Brandes I, et al. The burden of endometriosis: costs and quality of life of women with endometriosis and treated in referral centres. Hum Reprod 2012;27:1292-9.

9. Ballard K, Lowton K, Wright J. What's the delay? A qualitative study of women's experiences of reaching a diagnosis of endometriosis. Fertil Steril 2006;86:1296-301.

10. Hudelist G, Fritzer N, Thomas A, Nieues C, Oppelt P, Haas D, et al. Diagnostic delay for endometriosis in Austria and Germany: causes and possible consequences. Hum Reprod 2012;27: 3412-6.

11. Pugsley Z, Ballard K. Management of endometriosis in general practice: the pathway to diagnosis. Br J Gen Pract 2007;57: 470-6. 
12. Staal AH, van der Zanden M, Nap AW. Diagnostic delay of endometriosis in the Netherlands. Gynecol Obstet Invest 2016; 81:321-4.

13. Dmowski WP, Lesniewicz R, Rana N, Pepping P, Noursalehi M. Changing trends in the diagnosis of endometriosis: a comparative study of women with pelvic endometriosis presenting with chronic pelvic pain or infertility. Fertil Steril 1997; 67:238-43.

14. van der Zanden M, Nap AW. Knowledge of, and treatment strategies for, endometriosis among general practitioners. RBMO 2016;32:527-31.

15. Dancet EA, Apers S, Kremer JA, Nelen WL, Sermeus W, D'Hooghe $T M$. The patient-centeredness of endometriosis care and targets for improvement: a systematic review. Gynecol Obstet Invest 2014;78:69-80.

16. Geukens El, Apers S, Meuleman C, D'Hooghe TM, Dancet EAF. Patient-centeredness and endometriosis: definition, measurement, and current status. Best Pract Res Clin Obstet Gynaecol 2018;50:11-7.

17. Vercellini P. Management of endometriosis: moving toward a problem-oriented and patient-centered approach. Fertil Steril 2015;104:761-3.

18. Apers S, Dancet EAF, Aarts JWM, Kluivers KB, D'Hooghe TM, Nelen $W$. The association between experiences with patient-centred care and health-related quality of life in women with endometriosis. RBMO 2018;36:197-205.

19. Hirsh KW, Ladipo OA, Bhal PS, Shaw RW. The management of endometriosis: a survey of patients' aspirations. J Obstet Gynaecol 2001;21:500-3.

20. Burton C, Iversen L, Bhattacharya S, Ayansina D, Saraswat L, Sleeman D. Pointers to earlier diagnosis of endometriosis: a nested case-control study using primary care electronic health records. Br J Gen Pract 2017;67:e816-23.

21. Greene R, Stratton P, Cleary SD, Ballweg ML, Sinaii N. Diagnostic experience among 4,334 women reporting surgically diagnosed endometriosis. Fertil Steril 2009;91:32-9.

22. Glaser B, Strauss AL. The discovery of grounded theory: strategies for qualitative research. Chicago: Aldine Publishing Company; 1967.

23. Strauss A, Corbin J. Grounded theory methodology: an overview. Handbook of qualitative research. Thousand Oaks: Sage Publications; 1994.

24. Tong A, Sainsbury P, Craig J. Consolidated criteria for reporting qualitative research (COREQ): a 32-item checklist for interviews and focus groups. J Qual Health Care 2007;19:349-57.
25. Manderson L, Warren N, Markovic M. Circuit breaking: pathways of treatment seeking for women with endometriosis in Australia. Qual Health Res 2008;18:522-34.

26. Hadfield R, Mardon H, Barlow D, Kennedy S. Delay in the diagnosis of endometriosis: a survey of women from the USA and the UK. Hum Reprod 1996;11:878-80.

27. De Graaff AA, D'Hooghe TM, Dunselman GA, Dirksen CD, Hummelshoj L, Simoens S. The significant effect of endometriosis on physical, mental and social wellbeing: results from an international cross-sectional survey. Hum Reprod 2013;28: 2677-85.

28. Malterud K. The art and science of clinical knowledge: evidence beyond measures and numbers. Lancet 2001;358:397-400.

29. Malterud K. Qualitative research: standards, challenges, and guidelines. Lancet 2001;358:483-8.

30. Mays N, Pope C. Qualitative research in health care. Assessing quality in qualitative research. BMJ 2000;320:50-2.

31. Agarwal SK, Chapron C, Giudice LC, Laufer MR, Leyland N, Missmer SA, et al. Clinical diagnosis of endometriosis: a call to action. Am J Obstet Gynecol 2019;220:354.e1-12.

32. Taylor HS, Adamson GD, Diamond MP, Goldstein SR, Horne AW, Missmer SA, et al. An evidence-based approach to assessing surgical versus clinical diagnosis of symptomatic endometrixosis. Int J Gynaecol Obstet 2018;142:131-42.

33. Riazi H, Tehranian N, Ziaei S, Mohammadi E, Hajizadeh E, Montazeri A. Clinical diagnosis of pelvic endometriosis: a scoping review. BMC Women's Health 2015;15:39.

34. van der Zanden M, Teunissen TAM, van der Woord IW, Braat DDM, Nelen WLDM, Nap AW. Barriers and facilitators to the timely diagnosis of endometriosis in primary care. Fam Pract 2020;37: 131-6.

35. Shadbolt NA, Parker MA, Orthia LA. Communicating endometriosis with young women to decrease diagnosis time. Health Promot J Aust 2013;24:151-4.

36. Bush D, Brick E, East MC, Johnson N. Endometriosis education in schools: a New Zealand model examining the impact of an education program in schools on early recognition of symptoms suggesting endometriosis. Aust N Z J Obstet Gynaecol 2017;57: 452-7.

37. De Bie B. (V)erkenning van ervaringen leidt tot betere kwaliteit van zorg. NTOG 2016;129:2.

Supplementary Material: The online version of this article offers supplementary material (https://doi.org/10.1515/dx-2021-0043). 